\title{
Wrist Skin
}

National Cancer Institute

\section{Source}

National Cancer Institute. Wrist Skin. NCI Thesaurus. Code C52752.

The integumentary covering of one or both wrists. 